\title{
Análise das tendências de velocidades 2D em função da profundidade para detecção de anomalias de gás
}

Gleidson D. Ferreira (Petrobras), Frank C. Bulhões (Petrobras), Rogério A. Santos (Petrobras), Julio G. Carvalho (Petrobras), Arnaldo Tanaka (Petrobras), José Eduardo M. Lira (Petrobras).

Copyright 2018, SBGf - Sociedade Brasileira de Geofísica

Este texto foi preparado para a apresentação no VIII Simpósio Brasileiro de Geofísica, Salinópolis, 18 a 20 de setembro de 2018. Seu conteúdo foi revisado pelo Comitê Técnico do VIII SimBGf, mas não necessariamente representa a opinião da SBGf ou de seus associados. É proibida a reprodução total ou parcial deste material para propósitos comerciais sem prévia autorização da SBGf.

\section{Resumo}

On this work, we present a methodology to analyze the seismic velocity trends of Normal Moveout (NMO) 2D in relation to the two-way-time and the residual velocity generated by the misfit of these tendencies. Twenty-five lines of the Parnaíba Basin were analyzed. The main points of this methodology are: geological and stratigraphic representation, regional and semi-regional, between wells of the interval velocities estimated from NMO process; highlighting the relationship between interval velocities and reservoir, structural and petrophysical aspects, fluid migration paths and, finally, implantation of trend methods of seismic interval velocities with the wells influence. The application of the methodology shows that, from the use of the trends and their respective residues, it is possible the suggestion of fluids and the separation of processing artifacts.

\section{Introdução}

Um modelo de velocidade sísmica para ser o mais confiável possível deve ser: 1) geologicamente consistente; 2) utilizar dados de velocidade adequadamente amostrados; e 3) incorporar todas as informações de velocidade disponíveis, ponderando devidamente seus diferentes tipos, sísmicas e poços (Etris et al, 2001; Bulhões et al, 2014).

Pode-se entender um modelo de velocidade como geologicamente consistente a partir de sua adequada correlação com a estrutura das camadas: em regiões de análise sísmica envolvendo rochas compactadas isto normalmente significa ser conformante com a estrutura geológica, considerando contrastes litológicos, dobras e falhas. Em regiões de rochas com comportamento dúctil, as camadas podem simplesmente ser paralelas às estruturas da topografia ou batimetria e a variação da velocidade pode ser função principalmente do soterramento (Schultz, 1999). Em processos sísmicos de conversão de tempo para profundidade de múltiplas camadas, a seção é tratada em camadas separadas, cada uma com sua velocidade intervalar característica ou a partir de uma função velocidade que varie com a profundidade (Etris et al, 2001).

A velocidade sísmica em ambientes sedimentares tende a aumentar com maiores graus de compactação causada por sobrecarga das camadas mais rasas (Limpornpipat et al, 2012). No conjunto de dados utilizados na modelagem de velocidades (curvas profundidade-tempo de um perfil sônico vertical, check shot, VSP ou registro de sônico integrado) é possível obter uma velocidade instantânea, que é a variação de velocidade sobre pequenos incrementos de profundidade (Etris et al, 2001). Nos trabalhos de Al-Chalabi (1997 e 2001) e Ravve e Koren (2006) são apresentados uma gama de funções analíticas para descrever a variação da velocidade com a profundidade. O modo mais simples para descrever essa variação é representar a velocidade instantânea como função analítica linear da profundidade: $V(z)=V_{0}+k Z$, onde $\mathrm{V}(\mathrm{z})$ é a velocidade instantânea na profundidade $Z$, e $V_{0}$ e $k$ são a intercept e gradiente da função linear respectivamente. Dentro de uma determinada camada de rocha, a variação de velocidade com a profundidade pode ser descrita por um conjunto de valores $V_{0} e$ parâmetros $k$. Essas funções analíticas descrevem uma variação suave de velocidade com a profundidade, muito mais suave que as flutuações de alta frequência observadas em perfis sônicos. Na prática, nenhuma função analítica poderia representar a variação real de alta frequência da velocidade instantânea com a profundidade, pois o objetivo não é de descrever a geologia, mas sim verificar a tendência média da velocidade na unidade geológica em geral (Etris et al, 2001).

Estimativas de velocidade podem ser feitas a partir das variações de amplitude versus offset (AVO) que fornecem uma oportunidade de determinar a pressão de poro com a profundidade de dados sísmicos em seções transversais e volumes 3D (Chopra e Huffman, 2006).

A análise da velocidade é uma ferramenta utilizada para a inferência de anomalias de pressão de poros em 2D e 3D. Na maioria dos casos de análises de velocidades sísmicas para petróleo, as mudanças detectadas na velocidade intervalar são relacionadas à litologia, à porosidade, ao tipo de fluido nos poros e às mudanças de pressão dentro da coluna estratigráfica. Devido à compactação durante o soterramento das camadas geológicas, a velocidade intervalar das rochas siliciclásticas aumenta com a profundidade (Avseth et al, 2001). No entanto, existem anomalias na tendência de velocidade intervalar pela profundidade que coincidem com o início de zonas de pressões de poros anormalmente altas (Chopra e Huffman, 2006).

A pressão dos poros, ou pressão de formação, é definida como a pressão que atua nos fluidos no espaço poroso de uma formação. A ocorrência do fenômeno da pressão litostática se correlaciona bem, na maioria dos casos, com algumas características geológicas mapeáveis, tais como alterações de litologia, deformação de sedimentos e falhas.

As rochas com alta pressão de poros têm baixas velocidades. Isto é devido ao esforço sobre os poros que diminui a área de contato entre os grãos, diminuindo assim a eficiência da propagação da energia através do meio (Chopra e Huffman, 2006; Ebrom et al, 2007).

Surdam et al (2000) apresentam um exemplo do cálculo da tendência de velocidades realizado no campo de 
Jonah, localizado no condado de Sublette, estado de Wyamong, nos Estados Unidos. Este campo é um grande produtor de gás natural na bacia de Green River. Na Figura 1 é observada a análise da tendência e da anomalia estimada da velocidade a partir da leitura do perfil sônico. A anomalia é evidenciada através da retirada da tendência da velocidade obtida no perfil sônico, verificando-se uma anomalia de baixas velocidades.
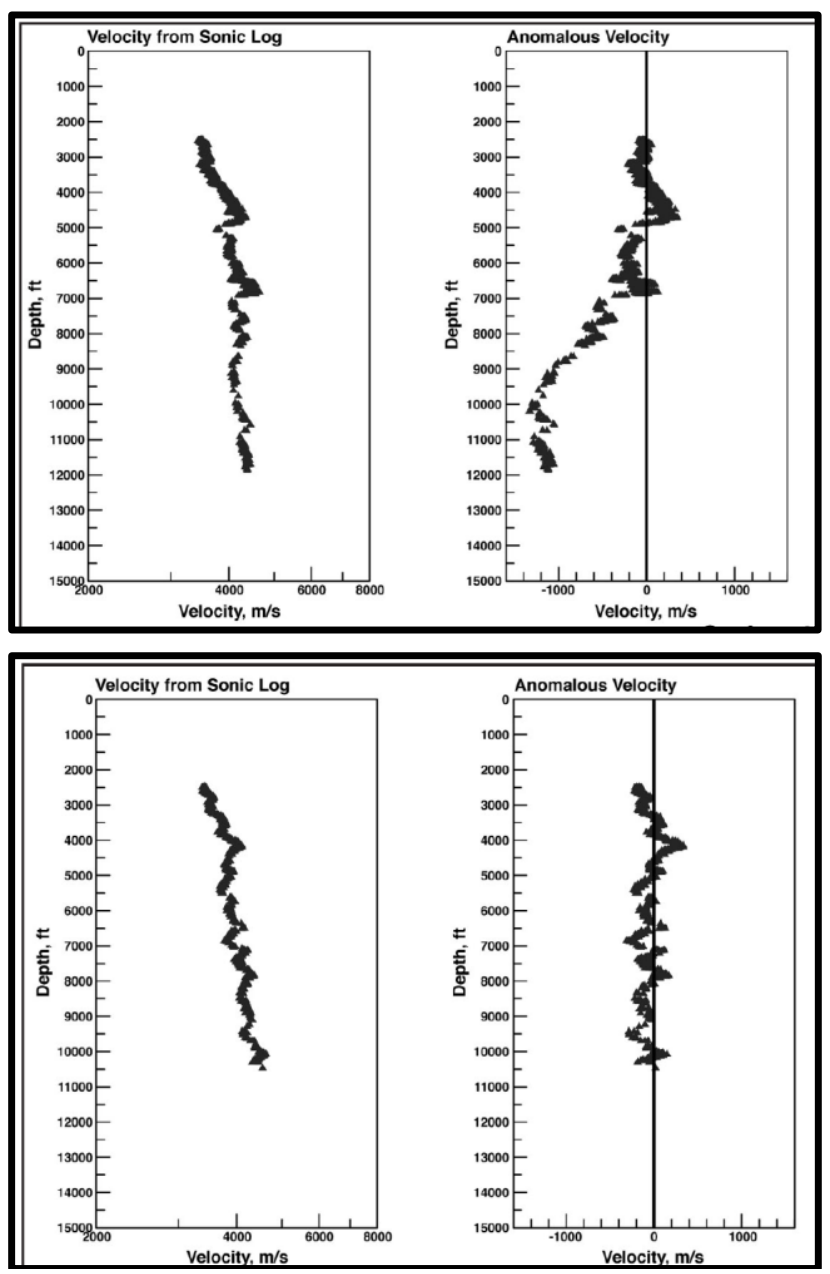

Figura 1 - Exemplo do cálculo da tendência de velocidades para obter a anomalia através da retirada desta tendência da velocidade do perfil sônico dos poços no campo de Jonah. Na figura superior (poço SHB 5-26), a retirada do gradiente regional de velocidade (tendência) da velocidade do perfil sônico, observa-se uma anomalia de baixas velocidades na profundidade entre $7.000 \mathrm{ft} e$ $12.000 \mathrm{ft}$. Na figura inferior (poço SHB 16-20), quando retirado o gradiente regional de velocidade (tendência) da velocidade do perfil sônico, observa-se que não há a presença de anomalias significativas. (Surdam et al. 2000).

\section{Metodologia}

A região de estudo localiza-se na bacia paleozoica intracratônica do Parnaíba, envolvendo uma área de aproximadamente $550.000 \mathrm{~km}^{2}$ (Figura 2). Os dados utilizados para o trabalho foram 33 poços, sete horizontes regionais, 25 linhas sísmicas $2 \mathrm{D}$ com dados de velocidade $\mathrm{NMO}$, oriundas do processamento, no domínio do tempo.

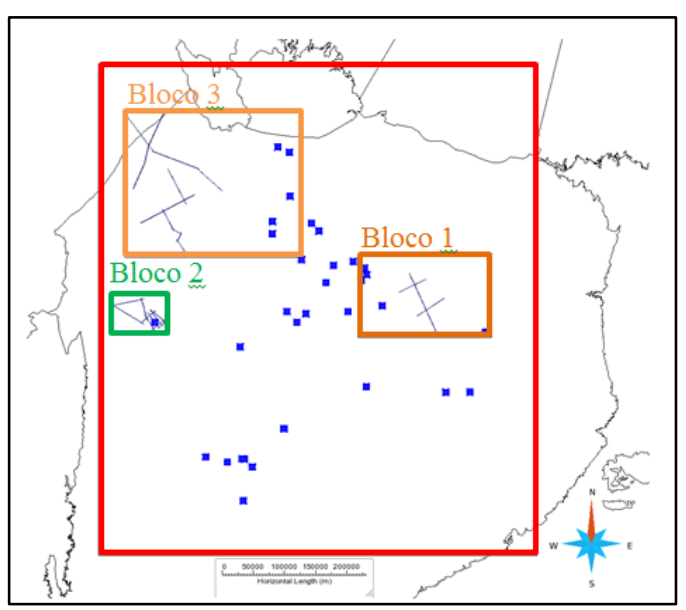

Figura 2 - Mapa da área de estudo na Bacia do Parnaíba com a distribuição das linhas $2 D$ e dos poços utilizados na análise das velocidades NMO. O retângulo vermelho indica a área de estudo e os outros retângulos menores indicam a localização dos blocos utilizados na segunda parte da metodologia.

O fluxo para extração da tendência das velocidades médias em função do tempo, cálculo e análise dos resíduos está representado na Figura 3. Nas primeiras etapas são extraídas as tendências da variação da velocidade, em função do tempo, usando regressão por mínimos quadrados nos dados de velocidade sísmica 2D e nas tabelas tempo-profundidade. Os resíduos calculados nos poços são indicativos da existência de anomalias referentes à presença de gás, caso esses valores sejam negativos quando comparados à tendência. A análise das tendências e resíduos nos poços são o principal insumo para calibração das velocidades $2 \mathrm{D}$ nas suas adjacências usando krigagem por deriva externa (Maul et al, 2013). As velocidades médias dos dados sísmicos utilizadas para estimação da tendência foram calculadas a partir das intervalares do processamento. A velocidade média dos poços foi obtida a partir das tabelas tempo-profundidade da amarração dos poços com os dados sísmicos.

A equação utilizada na regressão linear onde é obtido o trend de velocidades e a velocidade residual.

$$
\begin{gathered}
V=V(Z)=V_{0}+V_{\text {trend }}(Z)+V_{\text {res }} \\
V(z)=V_{0}+k Z+V_{\text {res }}
\end{gathered}
$$

Onde $\mathrm{V}_{0}$ é o intercept da velocidade; $\mathrm{k}$ é o gradiente de variação da velocidade com o tempo/profundidade; $V_{\text {res }}$ é o resíduo da velocidade. $\mathrm{O} \mathrm{V}_{\text {res }}$ pode ser descrito como:

$$
V_{\text {res }}=V_{\text {lito }}+V_{\text {fluido-poro }}+V_{\text {proc } / a q}
$$

Onde $\mathrm{V}_{\text {lito }}$ são as anomalias de fonte litológica, $\mathrm{V}_{\text {fluido-poro }}$ são anomalias devido à presença de fluidos e porosidade, $V_{\text {proc/aq }}$ são anomalias advindas de artefatos de aquisição e processamento. 


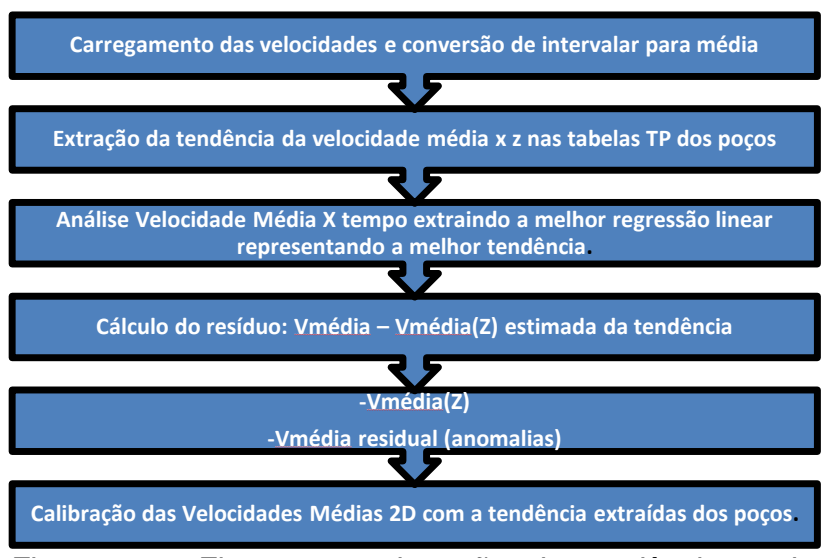

Figura 3 - Fluxo para obtenção da tendência e da velocidade residual dos poços.

\section{Análise das velocidades advindas das tabelas TP dos poços em função da profundidade}

As velocidades oriundas das tabelas tempo-profundidade dos poços disponíveis na área da Bacia do Parnaíba indicam que existe uma anomalia de velocidade representada pela velocidade residual $\left(V_{\text {res }}\right)$ (Figura 4).
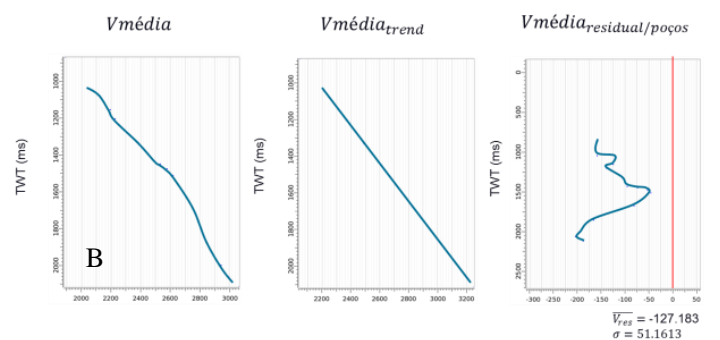

Figura 4 - $A$ velocidade média residual do poço $B$ apresenta uma anomalia negativa.

Realizou-se uma análise da velocidade média em função do tempo duplo, obtendo-se a função linear que melhor representasse a tendência da variação regional. Consequentemente, estimaram-se os resíduos dessa tendência regional em relação à velocidade oriunda das tabelas tempo-profundidade, dos poços (Figuras 5 e 6 ). Esses resíduos realçam os efeitos de alta frequência, os quais podem indicar variações locais na litologia, presença de rochas porosas e saturadas com fluidos e problemas relativos à perfilagem, como saltos de ciclos e arrombamentos no poço.

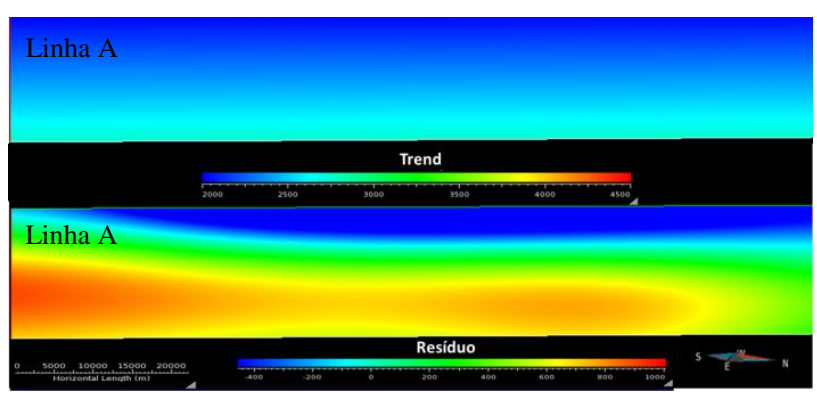

Figura 5 - Trend e resíduo da velocidade média dos poços extrapolado na linha $A$.

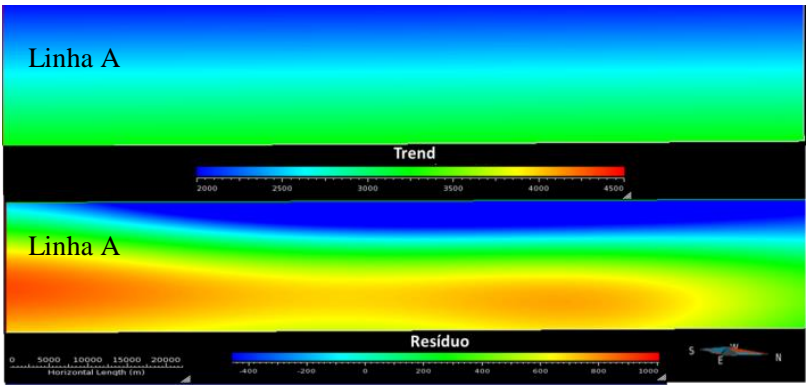

Figura 6 - Tendência e resíduo da velocidade intervalar dos poços extrapolados na linha $A$.

\section{Resultados}

Por meio de análise da correlação entre a velocidade média do poço $x$ tempo, obtiveram-se as curvas que melhor representassem a tendência da velocidade média de todos os poços da área de estudo, através de regressão linear, representada por reta (Figura 7a) e por regressão discreta (Figura 7b).
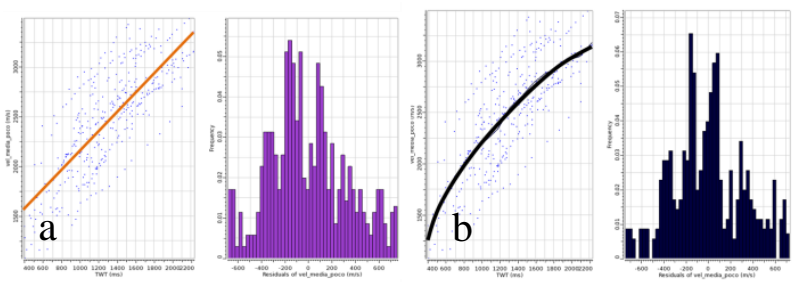

Figura 7 - a) Trend da velocidade média dos poços obtido através de uma regressão com uma curva contínua linear. b) Trend da velocidade média dos poços obtido através de uma regressão com uma curva contínua discreta à direita.

De modo a avaliar qual dos cenários de regressão, Figuras $7 \mathrm{a}$ e $7 \mathrm{~b}$, seria mais representativo, calibrou-se a velocidade intervalar sísmica da linha $2 \mathrm{D}$ com a velocidade intervalar obtida pelas tendências dos poços, usando krigagem com deriva externa. $O$ resultado dos dois cenários de calibração é apresentado na Figura 8. As anomalias resultantes no cenário de regressão discreta estão demasiadamente exageradas e não factíveis geologicamente. Por outro lado, na regressão linear a anomalia apresentada é mais sutil e geologicamente coerente e melhor se ajusta aos dados.

Vint $_{\text {calibrada/trend }}$ (Contínua) $\quad$ Vint $_{\text {calibrada/trend }}$ (Discreta)

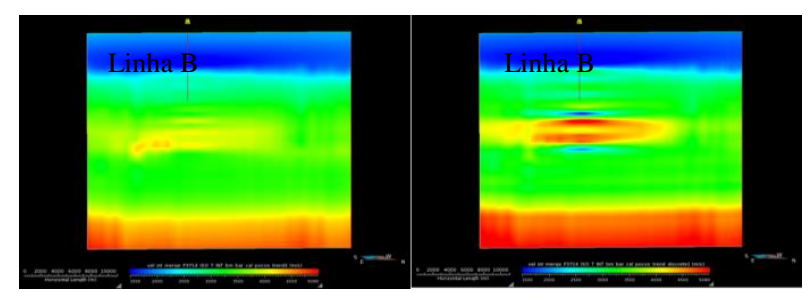

Figura 8 - velocidade intervalar calibrada com o trend dos poços da linha $B$ obtido através de uma regressão com uma curva linear e uma regressão com uma curva discreta.

Calibrou-se a velocidade média das linhas $2 \mathrm{D}$ com a velocidade média da tendência dos poços obtida da 
regressão linear, usando krigagem com deriva externa (Figura 9a). Converteu-se a velocidade média calibrada para intervalar de modo a analisar-se e ter um melhor controle de qualidade (Figura 9b). Na Figura 10 estão representados os gráficos da velocidade média dos poços na linha $A$ e o respectivo resíduo em função do tempo duplo na linha $A$.

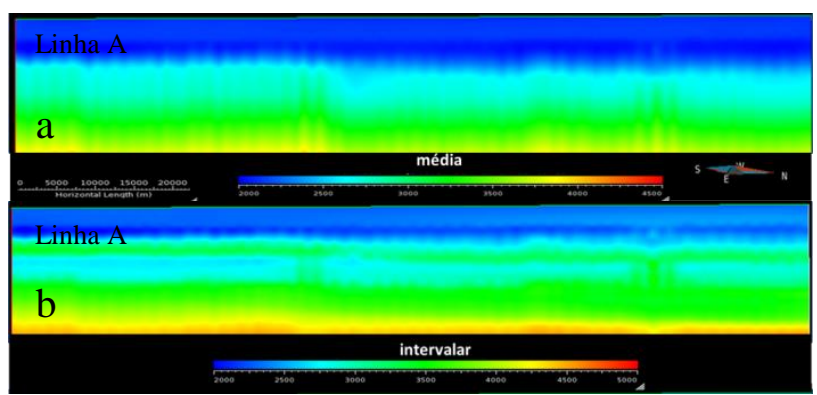

Figura 9- a) Velocidade média calibrada com o trend dos poços da linha $A$. b) Velocidade intervalar calibrada com o trend dos poços da linha $A$.

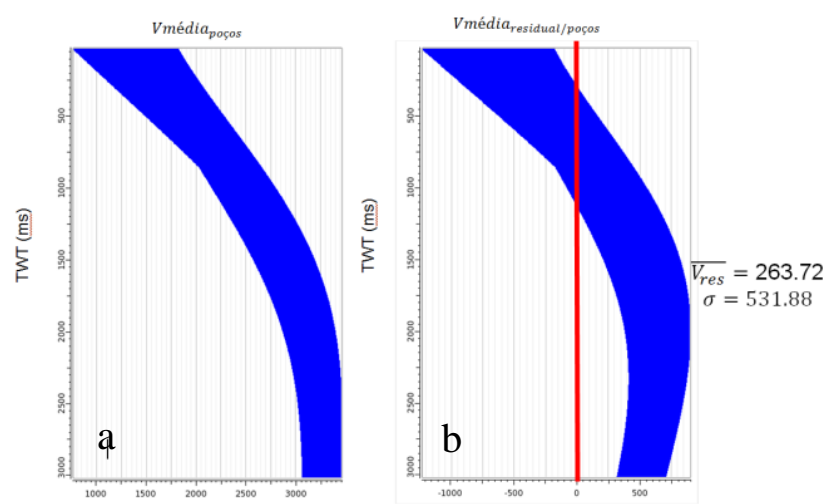

Figura 10 - a) Gráfico do tempo x velocidade média dos poços na linha $A$ e b) Gráfico do tempo $x$ velocidade média residual dos poços na linha $A$.

\section{Evolução da Metodologia}

Devido ao fato de existirem apenas levantamentos sísmicos 2D na Bacia do Parnaíba, regionalmente espaçados e irregularmente distribuídos, o mesmo ocorrendo com os poços, definiram-se três blocos para uma análise mais local e controlada da influência da tendência de velocidade média dos poços na velocidade média das linhas 2Ds. A distribuição e o limite dos blocos para análise local estão representados na Figura 3. O bloco 1 abrange uma área de aproximadamente 28.000 $\mathrm{km}^{2}$, com cinco poços e três linhas 2D. O bloco 2 tem extensão em torno de $6.000 \mathrm{~km}^{2}$, com sete linhas 2D e um poço. Por fim, o bloco 3 abrange aproximadamente $66.000 \mathrm{~km}^{2}$ com sete linhas sísmicas e cinco poços. Para cada bloco, realizou-se uma nova regressão da velocidade média do poço em função do tempo, obtendose uma curva que melhor representa a tendência da velocidade média dos poços através de um ajuste linear. Para cada bloco estimou-se a tendência da velocidade média dos poços e os respectivos resíduos. A velocidade média das linhas 2D foi calibrada com as tendências da velocidade média dos poços sendo posteriormente convertidas para velocidade intervalar, com objetivo de se fazer a análise e o controle de qualidade (Figuras 11 e 12).

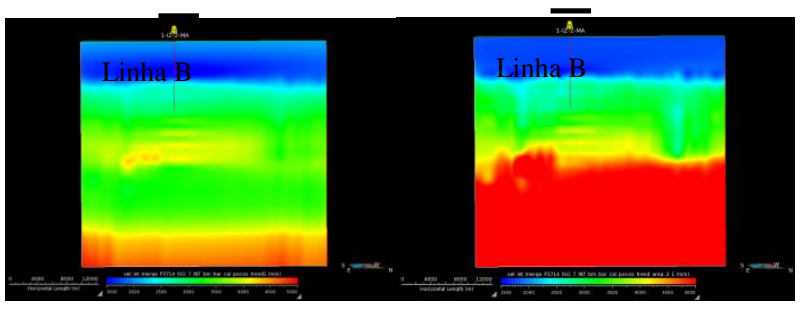

Figura 11- Velocidade intervalar calibrada com o trend dos poços da linha $B$ à esquerda e Velocidade intervalar calibrada com o trend dos poços da linha $B$ por blocos à direita (Bloco 2).

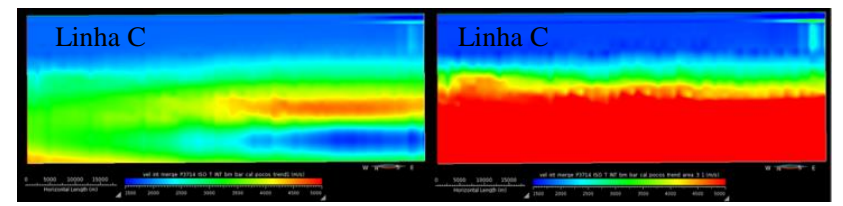

Figura 12 - Velocidade intervalar calibrada com o trend dos poços da linha $C$ à esquerda e Velocidade intervalar calibrada com o trend dos poços da linha $C$ por blocos à direita.

\section{Conclusões}

$\mathrm{Na}$ primeira parte da metodologia obteve-se a tendência e o resíduo das linhas $2 \mathrm{D}$ utilizando-se todos os poços da bacia (Figuras 5 e 6). Na Figura 4 observou-se que no poço $\mathrm{B}$, a velocidade média residual apresenta uma anomalia que pode indicar a presença de gás. Porém, optou-se por trabalhar com as velocidades das linhas 2D divididas em blocos para retirar a influência de poços mais distantes em todas a linhas.

$\mathrm{Na}$ segunda parte da metodologia, a velocidade média das linhas 2D calibrada com a tendência dos poços foi convertida para intervalar para análise e controle de qualidade na área dos blocos. Com isso, verificou-se que quando dividimos em blocos há uma maior influência dos poços mais próximos na velocidade das linhas 2D (Figuras 11 e 12). Na Figura 12 observam-se anomalias de alta e baixa, podendo ser devido a questões litológicas e presença de gás, respectivamente.

Esta metodologia pode ter as seguintes aplicações:

a) Análise multidisciplinar na inclusão da influência da tendência da velocidade média dos poços na velocidade média das linhas 2D, buscando-se uma correlação metodológica em áreas de bacias paleozoicas;

b) Avaliação volumétrica da análise bivariada (velocidade média do poço x tempo), para interpolação espacial de trends de velocidades média dos poços;

c) Análise $3 \mathrm{D}$ das velocidades médias das tendências dos poços, velocidade média residual dos poços e intervalar em função da tectônica e estratigrafia de blocos regionais;

d) Análise de sensibilidade da velocidade NMO à presença de gás não associado em situações de reservatórios tight e convencionais nas bacias interiores. 


\section{Agradecimentos}

Agradecemos à Petrobras por permitir a publicação deste trabalho. Agradecemos ao nosso gerente Francisco Aquino pelo apoio na publicação do trabalho.

\section{Referências}

Al-Chalabi, M., 1997. Time-depth relationships for multilayer depth conversion. Geophysical Prospecting no. 45: 715-720.

Al-Chalabi, M., 2001. The use of instantaneous velocity in uplift investigations. Geophysical Prospecting, no 49: 645655.

Avseth, P., Mavko, G., Dvorkin, J. and Mukerji, T., 2001. Rock Physics and Seismic Properties of Sands and Shales as a Function of Burial Depth. SEG Int'I Exposition and Annual Meeting. San Antonio, Texas September 914.

Bulhões, F. C. et. al, 2014. Fluxo para construção do Modelo de Velocidade Regional da Bacia de Campos. VI Simpósio Brasileiro de Geofísica da SBGF. Porto Alegre.

Chopra, S. and Huffman, A., 2006. Velocity determination for pore pressure prediction. Canadian Society of Exploration Geophysicists. Apr 2006, vol. 31, no. 04, view issue.

Ebrom, D., Albertin, M and Heppard, P., 2007. Can Pwave velocities be made more sensitive to pore pressure? SEG Technical Program Expanded Abstracts 2007.

Etris, E. L., Crabtree, N. J., Dewar, J., 2001. True Depth Conversion: More than a pretty picture. Canadian Society of Exploration Geophysicists, vol. 26 no. 09.

Faust, L. Y. (1951). Seismic Velocity as a function of depth and geologic time.

Fetter, M., Daltro, D., Lafferrieire, F. \& Souza JR., O. G., 2004. Filtragem de Velocidades Intervalares Através de Krigagem Fatorial. SIMGEF (Simpósio de Geofísica da Petrobras). Aracaju - SE.

Fruehn, J., F. Jones, I., Valler, V. Sangvai, P., Biswal, A., and Mathur, M., 2008. Resolving Near-Seabed Velocity Anomalies: Deep Water Offshore Eastern India. Geophysics, vol. 73, no. 5 September-October 2008; P. VE235-VE241.

Hoang, P., 2006, Rock Physics Depth Trend Analysis Using Seismic Stacking Velocity. Master of Science
Thesis. Norwegian University of Science and Technology. Department of Petroleum Engineering and Applied Geophysics. Trondheim, Norway.

Jones, I. F., 2012, "Absorption related velocity dispersion below a possible gas hydrate geobody. (accepted for publication in Geophysical Prospecting, as of 25/6/2012)

Kan, T. K. and Swanz, H. W., 2001. Geopressure prediction from automatically-derived seismic velocities. Geophysics, vol. 66, no. 6 (November-December, 2001): 1937-1946.

Limpornpipat, O., Laird, A., Tingay, M., Morley, C. K., Kaewla, C., Macintyre, H., 2012. Overpressures in the Northern Malay Basin: Part 2 - Implications for Pore Pressure Prediction. International Petroleum Technology Conference held in Bangkok, Thailand, 7-9 February 2012.

Maul, A., et al, 2013. Modelagem Geológica de Velocidades Sísmicas Utilizando o Gocad (Maio 2013) Material interno Petrobras.

Ravve, I. \& Koren, Z. Exponential asymptotically bounded velocity model: Part I - Effective models and velocity transformations. Geophysics, vol. 71, no. 3 May-June 2006; P. T53-T65.

Rosa, A. L. R., 2010. Análise do Sinal Sísmico - 1a edição. Rio de Janeiro - Sociedade Brasileira de Geofísica (SBGF).

Sayers, C. M., Johnsonz, G. M. and Denyer G., 2002, Predrill pore-pressure prediction using seismic data. Geophysics, vol. 67, no. 4 (July-August 2002): P. 12861292.

Surdam, R. C. Robinson, J., Jiao, Z. S., Boyd III, N. K. 2005. Delineation of Jonah Field Using Seismic and Sonic Velocity Interpretations. 2005 The Rocky Mountain Association of Geologists. Chaphter 13: 189-208.

van Dalfsen, W., Doornenbal, J.C., Dortland, S. \& Gunnink, J.L. 2006. A comprehensive seismic velocity model for the Netherlands based on lithostratigraphic layers. Netherlands Journal of Geosciences - Geologie en Mijnbouw vol. 85, no. 4: 277 - 292.

Vaz, P. T., Rezende, N. G. A. M., Filho, J. R. W., Travassos, W. A. S., 2007. Bacia do Parnaíba. B. Geoci. Petrobras, Rio de Janeiro, vol. 15, no. 2: 253-263.

Yamamoto, J. K., Landim, P. M. B., 2013. Geoestatística: conceitos e aplicações. São Paulo: Oficina de Textos, 